\title{
Differences between female and male patients with familial rheumatoid arthritis
}

\author{
S Laivoranta-Nyman, R Luukkainen, M Hakala, P Hannonen, T Möttönen,
} U Yli-Kerttula, J Ilonen, A Toivanen

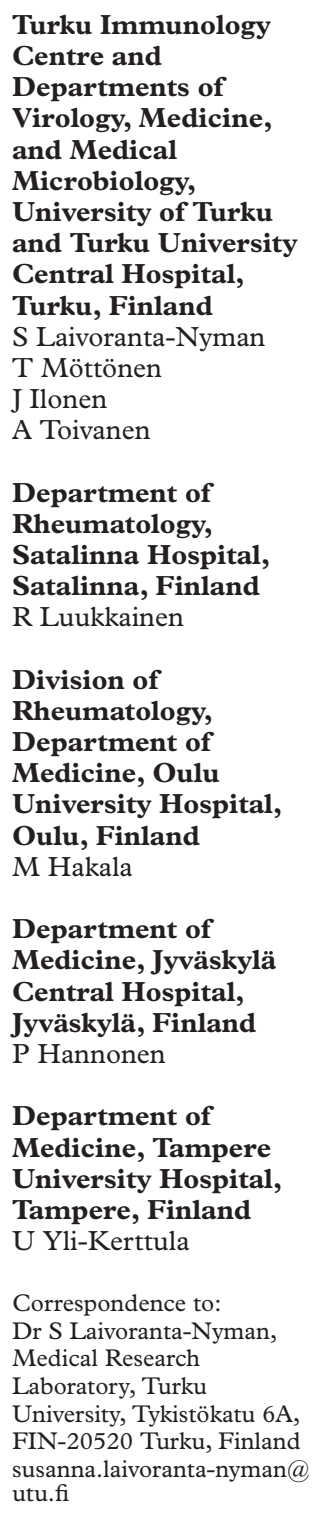

Accepted 5 September 2000

\begin{abstract}
Objective-To determine whether there are genetic differences between female and male patients with familial rheumatoid arthritis (RA).

Methods-45 men and 119 women from 78 families with RA who all had at least one first degree relative with RA were compared. HLA-DRB1 alleles were analysed, including DRB1`04 subtypes and associations of DRB ${ }^{\star} 04$ haplotypes with $D Q B 1{ }^{\star} 0301$ or $\mathrm{DQB} 1{ }^{\star} 0302$ alleles, the age of the patients at disease onset, the presence of rheumatoid factor (RF), joint erosions, and rheumatoid nodules.

Results-HLA-DRB1*13 allele (the subtype allele of DR6, reported to be protective against the development of RA) was found in $14 / 119(12 \%)$ of female but in none of the male patients $(p=0.036)$. The HLA-DR4 allele was found slightly more often in men than women patients with familial RA (31/45 (69\%) $v$ 75/119 (63\%), NS). Men were also more often RF positive than women $(44 / 45(98 \%) v 98 / 117$ $(84 \%) ; p=0.031)$. On the other hand, the mean age at onset of RA was significantly lower in the female group (40.4 years) than in men (46.6 years, $p=0.0044)$.

Conclusion-The results indicate that there is stronger genetic background in familial male than female patients with RA in the genetic susceptibility defined by the studied HLA antigens. However, the earlier age of onset of the disease in female group and the increased proportion of women with RA indicate that there are additional sex related predisposing factors enhanced in familial cases.

(Ann Rheum Dis 2001;60:413-415)
\end{abstract}

The ratio of the prevalence of women to men with rheumatoid arthritis (RA) has been consistently found to be around $3: 1,{ }^{1}$ though the probands from multicase families have been reported as often to be men as women. The observations that more women than men develop RA implies the presence of major susceptibility factor(s) other than HLA in female patients. $^{3}$ These might be hormonal or sex related genetic factors. ${ }^{4}$ Some studies suggest that the strength of the known HLA-DR4 association of RA is weaker in women than in men, ${ }^{4-6}$ but this has not been confirmed in other studies. ${ }^{3}$ A higher proportion of HLADR4 antigen in men than in women might be related to the presence of other susceptibility factors in women.
It has been suggested that families with an excess of affected men having a young age at onset may be the most informative in identifying the genetic factors either predisposing to o protecting from the development of RA. However, it is difficult to find a representative, sufficiently large number of families with an excess of affected men to carry out such studies. On the other hand, it seems that in some family studies the age at onset of RA was often younger in women than in men, though not lways reaching statistical significance. ${ }^{8-10}$ In the family study by Khan et al no difference in the age at onset of RA between familial male and female patients with RA was found.

One might expect that genetic factors accumulate in families with several members affected by RA. We could also show this for HLA-DR risk and protective alleles in our multicase family series, which we have now analysed for possible sex related differences. We compared the frequencies of HLA-DRB1 antigens, including HLA-DRB $1{ }^{\star} 04$ subtypes and DRB $1{ }^{\star} 04$ associated DQB 1 alleles, ages at the onset of disease, and the presence of theumatoid factor, bone erosions, or rheumatoid nodules in men and women.

\section{Patients and methods}

PATIENTS

We collected data on 78 Finnish families, in which at least two family members (first degree relatives to each other) had RA. Data of the index cases were collected from six different rheumatological units (Turku, Paimio, Satalinna, Tampere, Jyväskylä, and Oulu). Patients with RA who had at least one first degree relative with the disease were asked by questionnaire to participate in the study. The 78 families comprised 57 affected sib pairs, 16 parent-child pairs, and five sib trios. Additionally, there were also two children of two index cases affected and one mother of another index case affected in another sib pair. Rheumatoid factor (RF) status was not known for two female patients and in one male and one female patient the existence of rheumatoid nodules was unknown. Knowledge of erosions was missing in one female patient. Altogether 45 men and 119 women patients with familial RA, including index cases and affected family members, were included in this study.

All patients fulfilled the American College of Rheumatology (formerly the American Rheumatism Association) criteria for classification of RA ${ }^{11}$ Clinical documentation of all study patients included sex, age at disease onset (that is, at the time of diagnosis of RA), disease 
Table 1 Mean (SD) age of patients at disease onset (in years) in familial male and female $R A$ group

\begin{tabular}{llll}
\hline & Males $[n]$ & Females $[n]$ & $p$ \\
\hline All & $46.6(13.1)[45]$ & $40.4(14.3)[119]$ & 0.0044 \\
RF* positive patients & $46.7(13.2)[44]$ & $39.2(13.1)[98]$ & 0.0019 \\
RF negative patients & $45.0[1]$ & $46.4(18.9)[1]$ & NS \\
DR4 positive & $47.2(13.1)[31]$ & $39.7(14.9)[75]$ & 0.0057 \\
DR4 negative & $45.5(13.5)[14]$ & $41.6(13.3)[44]$ & NS \\
\hline
\end{tabular}

${ }^{\mathrm{RF}}=$ rheumatoid factor.

duration, serum RF status, and the presence of joint erosions or rheumatoid nodules. The patients were classified as seropositive if there had been one positive RF test at any time during the disease course. The test results for RF positivity and radiographs for erosions and positivity and radiographs for erosions and
existence of rheumatoid nodules were reexistence of rheumatoid nod
evaluated from the case records.

HLA TYPING

DNA was extracted from anticoagulated blood samples by a salting out method. The HLA-DR alleles were determined by a sequence-specific polymerase chain reaction (PCR) amplification. The low resolution genomic typing used could identify HLA antigens from DR1 to DR18 and also DR52 and DR53. When a single HLA-DRB1 allele could be amplified, the alleles were assumed to be homozygous. The presence of HLA-DQB1 alleles ${ }^{\star} 0301$ or ${ }^{\star} 0302$, or both, was defined by
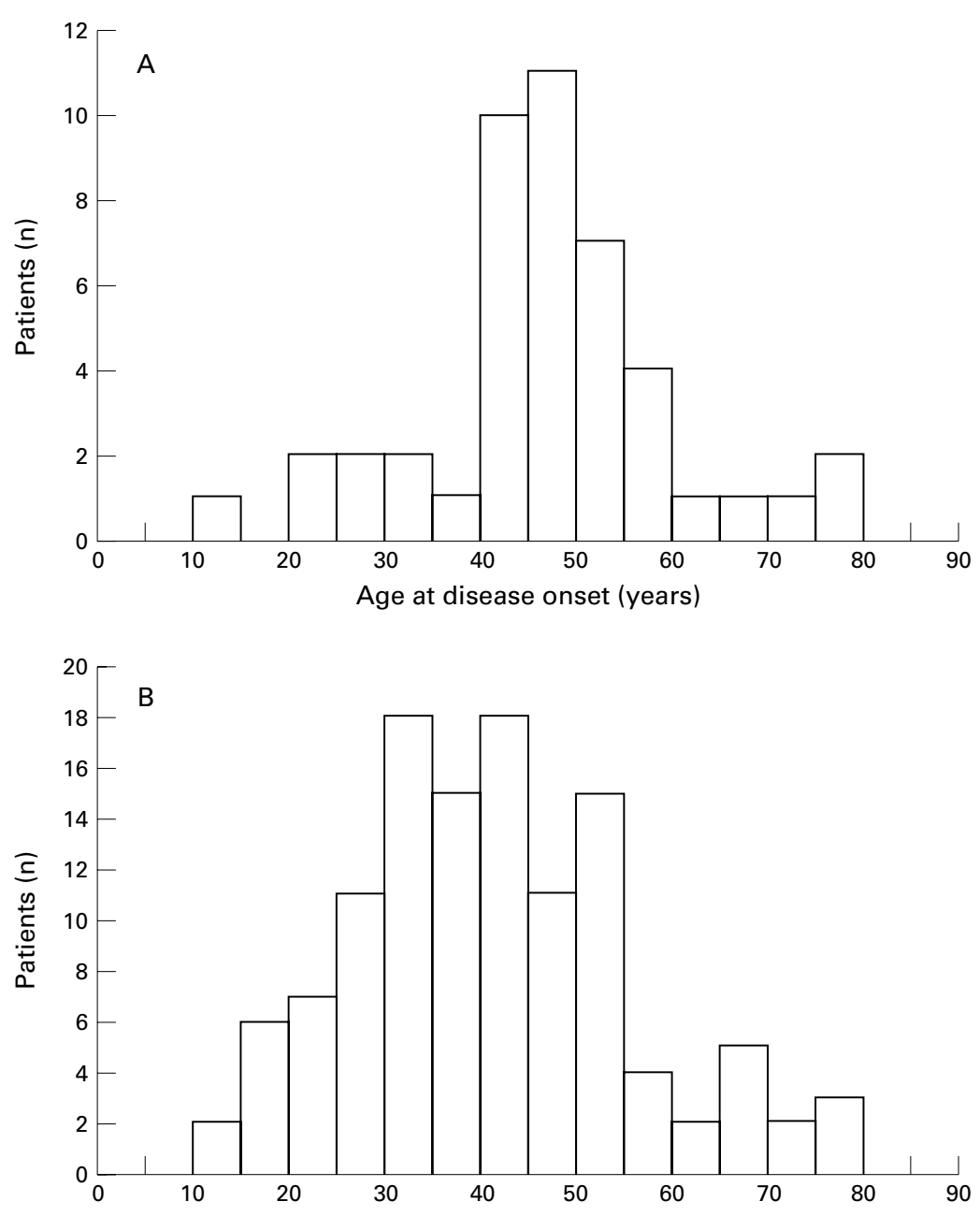

Age at disease onset (years) Figure 1 Age at disease onset in (A) male and (B) female patients with familial
rheumatoid arthritis. a method based on hybridisation of PCR products with a panel of lanthanide labelled oligonucleotide probes, and the HLA-DR4 subtypes were defined by a similar method designed for this purpose. ${ }^{10}$

STATISTICAL METHODS

Statistical analyses were performed with the $\chi$ test with continuity correction when differences in proportions of HLA frequencies, sex, $\mathrm{RF}$, rheumatoid nodule, or bone erosion status between the familial female and male patients were compared. Differences in the age f compared. Difference in the age at onset of disease and in the disease nalysed by a Mann-Whitney U test.

ETHICAL ISSUES

The study was accepted by the ethical committees of participating hospitals, and the samples were collected after informed consent.

\section{Results}

The male patients with familial RA were more often RF positive than female patients with familial RA. Only one seronegative man among 45 cases $(2 \%)$ was found compared with $19 / 117(16 \%)$ women $(p=0.031)$. Seronegative women were less often DR4 positive than RF ositive women. Thus, in particular, the particular, the number of seronegative HLA-DR4 negative women was higher, nine out of $117(8 \%)$ than the corresponding number in men $(1 / 45(2 \%)$, NS).

The HLA-DR4 antigen was slightly more common in familial men than women $(31 / 45$ $(69 \%) v 75 / 119(63 \%))$, but the difference did not reach statistical significance. The difference in the frequency of the so-called shared epitope (SE) common to DRB $1^{\star} 0401 /{ }^{\star} 0404 /$ epitope (SE) common to DRB $1{ }^{\star} 0401 /{ }^{\star} 0404 /$
${ }^{0} 0405 / \star_{0} 0408 / \mathrm{DR} 1$ and DR10 alleles, which ${ }^{\star} 0405 /{ }^{*} 0408 / \mathrm{DR} 1$ and DR10 alleles, which
was found in $40(89 \%)$ male and $99(83 \%)$ female patients, also did not reach significance. On the other hand, the HLA-DR13(6) antigen was found in 14/119 (12\%) women but in none of the male patients $(p=0.036)$. The frequencies of all other studied HLA-DR alleles were comparable in male and female patients with comparable in male and female patients with also made by analysing only probands from each family. Similar trends were found, though the differences were not statistically significant owing to the smaller numbers.

The HLA-DRB ${ }^{\star} 0401$ allele was the most common DR4 subtype in both groups, and no significant difference in the distribution of the subtypes was found between male and female patients with familial RA. We also determined the presence of HLA-DRB $1{ }^{\star} 04-\mathrm{DQB} 1{ }^{\star} 0301$ and $\mathrm{DRB} 1{ }^{\star} 04-\mathrm{DQB} 1{ }^{\star} 0302$ haplotypes in HLA-DR4 positive patients. Its presence was comparable in men and women (data not shown).

In men and women there were no differences in the proportions of patients with erosions $(36 / 45(80 \%)$ and $102 / 118(86 \%)$, respectively) or rheumatoid nodules (7/44 (16\%) and $21 / 118$ (18\%), respectively). Nevertheless the mean age at onset of RA was lower in the female group (table 1) and therefore the disease duration in them was also longer than 
in the male group (12.1 years $v 17.3$ years, $\mathrm{p}=0.092)$. The age difference at disease onset was statistically significant also when only subjects positive for RF and HLA-DR4 were compared. The distribution of the ages at disease onset is also shown in fig 1 , which clearly demonstrates the earlier onset of disease in the female group. Our patients included 35 patients from different generations (16 parentchild pairs, two affected children of probands, and one affected mother of a proband). To exclude the possible bias caused by these patients we analysed the results for the mean age of different patient groups at disease onset also including only patients in the same generation. In that comparison there were 38 male and 91 female patients with RA. All the results were essentially similar to those in the whole patient group $(\mathrm{p}=0.012$ for all patients in the same generation and $p=0.0061$ for those positive for RF and HLA-DR4).

Discussion

This study searched for evidence of possible genetic heterogeneity between male and female patients with familial RA. Interestingly, although it seems that familial male patients have more HLA associated genetic background, we found that the mean age of patients at disease onset was significantly lower in the female onset was significantly lower in the female
group. This suggests the presence of additional group. This suggests the presence of additional
genetic predisposing factor(s) in the familial genetic predisposing factor(s) in the familial female group. We think that those additional genetic factor(s) are common in the female patients with RA and, as suggested by Jaraquemada et al in 1986, explain the finding that more women than men develop RA despite the fact that both groups show a significant increase of DR4 frequency. ${ }^{3}$ The lower age at onset in the familial female group may indicate that the additional genetic predisposing factors are enhanced in familial cases at the same time as other predisposing factors (common to both sexes) are accumulating. In our previous study we did not find a lower age of onset in the nonfamilial female RA group. ${ }^{10}$

It has been suggested earlier that HLA genes are more important for the development of RA in men than in women. ${ }^{3}$ The decreased frequency of HLA-DRB $1^{\star} 13$ and slightly increased frequency of the HLA-DRB $1{ }^{\star} 04$ in our male patients with familial RA as compared with the female patients is in accordance with this hypothesis. The numbers are still small and the $\mathrm{p}$ value for the difference in $\mathrm{DRB} 1^{\star} 13$ is significant only when calculated without correction for the number of comparisons made. However, we detected a protective effect of HLA-DRB $1{ }^{\star} 13$ in RA in both familial and sporadic cases, ${ }^{10}$ and this has also been found in several earlier studies, though not always reaching a statistical significance. ${ }^{12-14}$ The effect might also be due to the associated DQ alleles, which have been suggested to be of primary importance in genetic protection against the disease. . $^{15}$

This study showed an increased frequency of RF positivity in the male patients compared with the female patients; the same phenomenon has been reported earlier in patients with sporadic RA. ${ }^{4}$ The reason for this higher incidence of RF in male patients with RA is not known but may reflect the effect of the difference in the genetic background of the sexes in the pathogenesis of RA. There may exist a seronegative non-HLA associated disease, more often affecting women, which would have profound differences in its aetiopathogenesis. We thank Ms Terttu Laurén, Ms Ritva Suominen, Ms Mia
Karlsson, Ms Karoliina Karlson, and Ms Leena Kivistö for skil-
ful technical help. This study was supported by the Turku University Foundation
and EVO grant of Turku University Central Hospital.

1 Lawrence JS. Heberden Oration, 1969. Rheumatoid 1970;29:357-79. or nurture? Ann Rheum Dis 2 Lynn AH, Kwoh CK, Venglish CM, Aston CE, Chakravarti
A. Genetic epidemiology of rheumatoid arthritis. Am J

3 Jaraquemada D, Ollier W, Awad J, Young A, Silman A, Roit IM, et al. HLA and rheumatoid arthritis: a combined
analysis of 440 British patients. Ann Rheum Dis 1986;45: $627-36$.

4 Weyand CM, Schmidt D, Wagner U, Goronzy JJ. The influence of sex on the phenotype of rheumatoid arthritis Arthritis Rheum 1998;41:817-22.
Maeda H, Juii T, Mitsui H, Sonozaki H, Okitsu K. HLA MR4 and rheumatoid arthritis in Japanese people. An Rheum Dis 1981;40:299-302.
6 Ohta N, Nishimura YK, Tanimoto K, Horiuchi Y, Abe C, patients with rheumatoid arthritis. Hum Immunol 1982;5. 123-32.

7 Khan MA, Yamashita TS, Reynolds TL, Wolfe F, Kha familial rheumatoid arthritis. Tissue Antigens 1988; in 254-8.

8 Sanders PA, Grennan DM, Dyer PA, Thomson W, deLang G. A comparison of clinical and immunogenetic feature 1987;14:718-22.

9 Deighton CM, Walker DJ. What factors distinguis probands from multicase rheumatoid arthritis same sex
sibships from sporadic disease? J Rheumatol 1992;19:237-

41.
10 Laivoranta-Nyman S, Möttönen T, Luukkainen R, Hakala
M Y Yli-Kertula M, Yli-Kerttula U, Hannonen P, et al. Immunogenetic di reucistoid arthritis. Ann Rheum Dis 2000;59:173-7.
rheumatos

1 Arnett FC, Edworthy SM, Bloch DA, McShane DJ, Fries tion 1987 revised criteria for the classification of Associatoid arthritis. Arthritis Rheum 1988;31:315-24.

12 van Zeben D, Hazes JMW, Zwinderman AH, Cats A, Schreuder GMT, DNW, ZJ, et al. Association patients with rheumatoid arthritis. Results of a followu study. Arthritis Rheum 1991;34:822-30.

(DE, Ollier WER Khan MA, et al Investigating the HLA compont

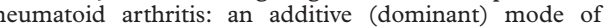

4 Widitanis S Murata N Todat

Nishimura Y, et al. The relationship between HLA-DRB alleles and disease subsets of rheumatoid arthritis Japanese. Br J Rheumatol 1997;36:630-6.

Zanelli E, Huizinga TWJ, Guerne P-A, Vischer TL, Tiercy
$\mathrm{J}-\mathrm{M}$, Verduyn W, et al. An extended HLA-DQ-DR haplotype rather than DRB1 alone contributes to RA pre-
herd a 